\title{
A Review on Antenna Radiation Pattern Synthesis
}

\author{
Meenakshi L Rathod ${ }^{1}$, Dr. A. Meera ${ }^{2}$. \\ ${ }^{I}$ Dr.AmbedkarInstitute of technology, Bangalore, India \\ ${ }^{2}$ B.M.S.College of Engineering, Bangalore, India
}

\begin{abstract}
In recent communication systems side lobe level reduction has become one of the important issue. For applications like digital beam forming it is one of the important factor because it reduces the effect of interference arriving outside the main lobe. This reduction in the interference increases the capacity of the communication systems.

This paper presents the different methods for reducing the side lobe level and increasing the directivity. In several occasions a single element antenna is unable to meet the gain or highly directive radiation pattern requirements especially suited for long distance communication. Antenna arrays are formed to circumvent such problems by combining many individual antenna elements in certain electrical and geometrical configurations. The purpose of this research is to synthesize the radiation pattern for the design of different phased array structures considering the optimization process by using the natural computing to achieve the higher directivity with minimizing the side lobe level.
\end{abstract}

Keywords: Side lobe level (SLL), Antenna array, Differential Evolution (DE).

\section{Introduction}

In several occasions a single element antenna is unable to meet the gain or highly directive radiation pattern requirements especially suited for long distance communication. Antenna arrays are formed to circumvent such problems by combining many individual antenna elements in certain electrical and geometrical configurations. Antenna arrays have been widely used in diverse applications including radar, sonar, radios, and third generation wireless communication systems. Wireless communication technologies have experienced fast growth in recent years. The latest mobile devices offer multi-bandwidth services and to enable this, new technologies have to be developed. Spatial processing is considered the last frontier in the battle for improved cellular systems and smart antennas are emerging as the enabling technique. The use of adaptive antenna arrays in mobile handsets can help eliminate co-channel interference and multi-access interference among other problems. These breed of antennas are able to radiate power towards a desired angular sector, thus, avoiding interference with undesired devices.

The primary design objective of antenna array geometry is to determine the positions of array elements that jointly produce a radiation pattern that matches the desired pattern as closely as possible . In recent past design of non-uniformly spaced linear arrays received a great amount of attention from researchers working in electromagnetic optimization problems. The computational drawbacks of existing numerical methods have forced the researchers all over the world to rely on metaheuristics algorithms founded on simulations of some natural phenomena to solve antenna problems. Since the classical derivative-based optimization techniques are prone to getting trapped in local optima and are strongly sensitive to initialization, many modern metaheuristics were tried to achieve optimized Side Lobe Level (SLL).The antenna arrays optimization for improving performance represents an open line of research in the antenna design field.

\section{Review of the previous work}

An efficient optimization method, the differential evolution algorithm (DEA), is proposed for the synthesis of uniform amplitude arrays of two classes, i.e., unequally spaced arrays with equal phases and unequal phases [1]. The DEA is applied to develop a computationally efficient synthesis tool for the design of uniform amplitude, unequally spaced arrays. Phase-only synthesis and the synthesis of uniformly exited unequally spaced arrays (position only synthesis) are compared and it is seen that, by using the unequal spacing, the number of array elements can be significantly reduced for attaining reduced side lobe levels. From the DEAbased synthesis of unequally spaced arrays with uniform amplitudes and unequal phases, it is found that a tradeoffs exists between the size of the unequally spaced arrays and the range of phases for the same radiation characteristics. The proposed synthesis technique using uniform amplitudes, unequal spacing, and unequal phases (position-phase synthesis) not only decreases the size of the array for the same side lobe level compared to both the phase-only synthesis and position-only synthesis but also retains their advantages [1]. Improved array efficiency and decreased side lobe levels have been achieved in the design of the pencil beam array using DEA-based position-phase synthesis compared to the phase-only synthesis. 
A synthesis method based on differential evolution algorithm is proposed [2] for conformal antenna arrays in the presence of platform. Differential evolution algorithm is used to solve the practical synthesis of the conformal array antenna. With the desired phase weights determined by the scan angle and geometry of the array, the amplitude weights of elements are optimized by differential evolution algorithm to reduce the level of side-lobes. Both the effect of platform on radiation pattern of conformal array and the mutual coupling between the elements are taken into account by calculating the antenna array and platform simultaneously using the method of moment. The coupling of antennas and their platform can be taken into account by using of moment method. The storage and computation needed by MoM are reduced by the application of AIM [2].

The improved antenna pattern synthesis based on particle swarm optimal algorithm is presented [3]. As an example, the design goals of normalized antenna pattern : the main lobe width of $20^{\circ}$, requiring $0^{\circ}, 5^{\circ}, 10$ $\circ, 20^{\circ}, 40^{\circ}, 55^{\circ}, 60^{\circ}, 70^{\circ}, 80^{\circ}$ generates - 90dB zero trap, the side lobe level below-50dB (using absorbing boundary conditions). Simulation for this method is used for more than 20 particles, there are 130 optimization iterations. Through particle swarm optimization algorithm simulation, the ultimate incentive value of the array element optimization results are calculated. From the simulation, it is seen that the algorithm applied to the array antenna pattern synthesis meets the precision and stability requirements [3].

The design problem of non-uniform circular antenna arrays for the best optimal performance can be solved with the help of seeker optimization algorithm [4]. The seeker optimization algorithm efficiently computes the parameters of non-uniform circular antenna array to generate the near optimal radiation pattern. The algorithm is used to determine an optimum set of current excitation weights and antenna inter-element separations that provide radiation pattern with maximum reductions of side lobe level (SLL) and first null beamwidth (FNBW). Circular array antennas lying on $\mathrm{x}$-y plane are assumed. The same algorithm is applied successively on circular arrays of 8,10 and 12 elements. For design of non-uniform circular antenna arrays using the same algorithm provides considerable reductions of side lobe level and first null beamwidth as compared to the uniform case and some published works as well. Also, array patterns obtained by the SOA are generally better than those presented before [4].

The design of nonuniformly spaced linear array antennas is done using Particle Swarm Optimization method [5]. The purpose of this is to match a desired radiation pattern and improve the performance in terms of sidelobe level. Two approaches are considered: in the first, the design of element placement with the constraint of array length being imposed is performed. The second is based on element position perturbation starting from a uniform element distribution. The particle swarm optimization technique, which is a relatively new evolutionary optimization algorithm based on random search principle, has proved to be a powerful and an effective tool for designing the optimum. Both design approaches illustrate almost identical results. However, the first approach puts a further constraint on the array length which is a practical choice. The designs satisfy the requirements of wireless communications systems and present an optimal geometry of antenna system that provides minimal signal-of-non-interest (interferers) reduction capability and enhances the signals-of-interest (very high directivity) [5].

Designing circular antenna arrays with minimum SLL, maximum directivity, and also minimum size of the circumference is a challenging optimization problem in electromagnetism. In this paper an optimization method for design of non-uniform, planar, and circular antenna arrays with minimum side lobe levels for a specific first null beamwidth and also a minimum size of the circumference is discussed [6]. The two metaheuristics methods are the Invasive Weed Optimization (IWO) and the Differential Evolution (DE). IWO is a derivative-free real parameter optimization technique that mimics the ecological behavior of colonizing weeds and then modifying the classical IWO by incorporating the difference vector based mutation schemes from the realm of DE.The simulation results using these methods indicates, the hybrid DIWO could comfortably outperform PSO, DE, and GA over 8, 10, and 12 element array design problems based on metrics such as average final accuracy, best obtained design figures of merit (like SLL, directivity, circumference size in terms of wavelength), convergence speed, and robustness, in a statistically significant manner. All these factors together (not just a single factor separately or a combination of some of the factors) are considered for optimal results in this work [6].

Design of non-uniform circular antenna arrays is one of the important optimization problems in electromagnetic domain. While designing a nonuniform circular array the goal of the designer is to achieve minimum side lobe levels with maximum directivity [7]. In contrast to the single-objective methods that attempt to minimize a weighted sum of the four objectives considered here, in this paper [7], the four distinct objectives that are to be optimized simultaneously in a multi-objective (MO) framework using one of the best known Multi-Objective Evolutionary Algorithms (MOEAs) called NSGA-II. This MO approach provides greater flexibility in design by producing a set of final solutions with different trade-offs among the four objective from which the designer can choose one as per requirements. This MO framework provides immense advantages to the designer as the MOEAs generate the Pareto optimal set from which the designer can choose a desired solution and helps in finding the right balance between the four objectives. The simulation results 
indicate the best compromise solution obtained by NSGA-II algorithm could comfortably outperform the best results obtained with the traditional single-objective DE and PSO algorithms over the 8 element array design problem demonstrating the efficiency as well as effectiveness of the MO framework over the single objective one. NSGA-II is also able to achieve minimum SLL and maximum directivity among the competitors [7].

In this paper optimal side lobe reduction is achieved for time modulated linear antenna arrays with optimized uniform inter-element spacing and non-uniform excitations using an evolutionary optimization method RGA (Real Coded Genetic algorithm) [8]. The side lobe level of time modulated linear array can be reduced significantly by optimal rearrangement of static excitation amplitudes and inter-element spacing of each element by RGA. Experimental results of this paper reveal that the optimal design of non-uniformly excited time modulated linear antenna arrays with optimal inter-element spacing offers a considerable SLL reduction with respect to corresponding time modulated uniform linear arrays with uniform inter-element spacing of $\lambda / 2$. For the time modulated linear antenna array sets having 12, 16 and 20 elements, SLLs have reduced to -23.66 $\mathrm{dB},-20.72 \mathrm{~dB}$ and, $-18.26 \mathrm{~dB}$, respectively, against $-5.424 \mathrm{~dB},-5.261 \mathrm{~dB}$ and $-5.148 \mathrm{~dB}$, respectively, of corresponding uniform time modulated linear arrays with a very little change in BWFN [8].

A new and very speedy synthesis method for linear array antennas with periodic element spacing is based on Taguchi's method [9]. A set of phase shift weights are generated in order to steer the beam towards any desired direction and avoiding interference. These weights were optimized in order to maximize the power of the main lobe at a desired direction while keeping nulls towards interferers. The results of Taguchi's method are validated by using rectangular patch antenna and simulated by software CST2009 (CST microwave studio) [9].

In this paper, an optimal radiation pattern is obtained for a linear antenna array using the particle swarm optimization technique [10]. In this paper, the Particle Swarm Optimization method was used to obtain a set of phase shift weights that configure a linear antenna array. These weights were optimized in order to maximize the power of the main lobe at a desired direction while keeping nulls towards interferers. A comparison with a Genetic Algorithm was studied and the results of 1000 experiments show that the PSO achieves better and more consistent radiation patterns than those of the GA. It was also observed that the total number of fitness function evaluations is lower for the PSO, which suggests an advantage in terms of performance as the function evaluation tends to have higher computational cost [10].

Design of non-uniform linear antenna arrays is one of the most important electromagnetic optimization problems of current interest [11]. In this paper, an adaptive Differential Evolution (DE) algorithm has been used to optimize the spacing between the elements of the linear array to produce a radiation pattern with minimum side lobe level and null placement control. DE is arguably one of the best real successfully found out the optimal array element location so that the array-pattern has either suppressed side lobe or null direction or both [11].

\section{References}

[1]. Dhanesh G. Kurup, Mohamed Himdi, and Anders Rydberg," Synthesis of Uniform Amplitude Unequally Spaced Antenna Arrays Using the Differential Evolution Algorithm" IEEE Transactions on Antennas and Propagation, Volume: 51 , Issue: 9 , 2003 , Page(s): 2210 - 2217

[2]. Jing-Li Guo and Jian-Ying Li , "Pattern Synthesis of Conformal Array Antenna in the Presence of Platform Using Differential Evolution Algorithm" " IEEE Transactions on Antennas and Propagation, Volume: 57 , Issue: 9, 2009, Page(s): 2615 - 2621

[3]. Duan Li ; Coll. of Inf., Beijing Union Univ., Beijing, China ; Xue Yong-yi ; Zhao Yi-song, “Antenna pattern synthesis based on the particle swarm optimal algorithm “, IEEE Proc. Future Computer and Communication (ICFCC), 2010 2nd International Conference on (Volume:2) Page(s):V2-292 - V2-295

[4]. Gopi Ram, Durbadal Mandal, Sakti Prasad Ghoshal, and Rajib Ka1 "Design of Non-uniformly Weighted and Spaced Circular Antenna Arrays with Reduced Side Lobe Level and First Null Beamwidth Using Seeker Optimization Algorithm", (C) Springer International Publishing Switzerland 2013, pp. 35-46.

[5]. Abdelmadjid Recioui, "Sidelobe Level Reduction in Linear Array Pattern Synthesis Using Particle Swarm Optimization", (C) Springer Science+Business Media, LLC 2011.pp. 497-512.

[6]. Basak, A. Pal, S. ; Das, S. ; Abraham, A." Circular Antenna Array Synthesis with a Differential Invasive Weed OptimizationAlgorithm" IEEE Proc. Hybrid Intelligent Systems (HIS), 201010 th International Conference , Page(s): 153 - 158.

[7]. Subhrajit Ro1, Sk. Minhazul Islam, Saurav Ghosh, Shizheng Zhao,Ponnuthurai Nagaratnam Suganthan, and Swagatam Das, "Nonuniform Circular-Shaped Antenna Array Design and Synthesis - A Multi-Objective Approach" Springer-Verlag Berlin Heidelberg 2011, SEMCCO 2011, Part II, LNCS 7077, pp. 223-230, 2011.

[8]. Gopi Ram Hardel, Durbadal Mandal, Sakti Prasad Ghoshal, and Rajib Kar, "Minimization of Side Lobe of Optimized Uniformly Spaced and Non-uniform Excited Time Modulated Linear Antenna Arrays Using Genetic Algorithm" Springer-Verlag Berlin Heidelberg 2012,pp. 451-458.

[9]. Amor Smida, Rihda Ghayoula and Ali Gharsallah," Phase-only adaptive nulling with Taguchi's Method for antenna array synthesis", American Journal of Applied Sciences, 2012, 9 (11), 1833-1839.

[10]. Zuniga, V. ; Erdogan, A.T. ; Arslan, T. , "Adaptive radiation pattern optimization for antenna arrays by phase perturbations using particle swarm optimization”, IEEE Proc. Adaptive Hardware and Systems (AHS), 2010 NASA/ESA, Page(s): 209 - 214.

[11]. Chowdhury, A. ; Giri, R. ; Ghosh, A. ; DAs, S. ; Abraham, A. ; Snasel, V. "Linear Antenna Array Synthesis Using Fitness-AdAPtive DifFERENTIAL Evolution AlgorithM” IEEe Proc. Hybrid Evolutionary COMPUTATION (CEC), 2010 IEEE Congress, 2010 InTERnATIONAL CONFERENCE , PAGE(S):1- 8. 